\title{
Effective dynamics of self-gravitating extended objects
}

\author{
S.Ansoldi* \\ Dipartimento di Fisica Teorica dell'Università, \\ Strada Costiera 11, 34014-Trieste, Italy, \\ A.Aurilia ${ }^{\dagger}$ \\ Department of Physics, California State Polytechnic University \\ Pomona, CA 91768, USA, \\ R.Balbinot ${ }^{\ddagger}$ \\ Dipartimento di Fisica dell'Università, \\ Istituto Nazionale di Fisica Nucleare, Sezione di Bologna, \\ via Irnerio 46, 40126-Bologna, Italy, \\ E.Spallucci ${ }^{\S}$ \\ Dipartimento di Fisica Teorica dell'Università, \\ Istituto Nazionale di Fisica Nucleare, Sezione di Trieste, \\ Strada Costiera 11, 34014-Trieste, Italy,
}

*E-mail address: ansoldi@vstst0.ts.infn.it

†E-mail address: aaurilia@csupomona.edu

${ }^{\ddagger}$ E-mail address: balbinot@bologna.infn.it

$\S_{\text {E-mail address: spallucci@vstst0.ts.infn.it }}$ 


\begin{abstract}
We introduce an effective Lagrangian which describes the classical and semiclassical dynamics of spherically symmetric, self-gravitating objects that may populate the Universe at large and small (Planck) scale. These include wormholes, black holes and inflationary bubbles. We speculate that such objects represent some possible modes of fluctuation in the primordial spacetime foam out of which our universe was born. Several results obtained by different methods are encompassed and reinterpreted by our effective approach. As an example, we discuss: i) the gravitational nucleation coefficient for a pair of Minkowski bubbles, and ii) the nucleation coefficient of an inflationary vacuum bubble in a Minkowski background
\end{abstract}




\section{INTRODUCTION}

According to some current ideas in cosmology, in particular the chaotic inflationary scenario [1], the Universe may consist of infinitely many self-reproducing bubbles which are continuously nucleated quantum mechanically. Some of them expand and look like a Friedmann universe, others may collapse to form black holes, and some may be connected by wormholes. In principle, this dynamical network may exist at any scale of distance, or energy; at the Planck scale of energy, it is expected to arise from gravitational fluctuations which induce a foam-like structure over the spacetime manifold. Some work on the dynamics of the constituents of this network has already appeared in the literature [2] 6], but the results so far obtained are not always consistent and seem to be model dependent. As a matter of fact, the very notion of spacetime foam, introduced by Wheeler nearly forty years ago [7], has periodically come under close scrutiny in the intervening years. The point of fact is that a complete quantum theory of the above processes is still beyond our reach, and one must come up with some viable alternative.

The aim of this communication is to suggest an effective approach to the dynamics of spherically symmetric, self-gravitating objects that may arise, evolve and die in the spacetime foam. Among these "objects", somehow, there is the universe in which we live, and therefore the study of such fluctuations hardly needs a justification. In order to give some analytical substance to this qualitative picture, we envisage the spacetime foam as an ensemble of vacuum bubbles, or cells of spacetime, each characterized by its own geometric phase and vacuum energy density. In principle each cell may behave as a black hole, a wormhole, an inflationary bubble, etc., depending on the matching conditions on the neighboring cells [8]. Thus, a useful starting point for our present discussion, is the general matching equation between the internaland external metrics of a self-gravitating, spherically symmetric bubble [9]

$$
\sigma_{\text {in }} R \sqrt{1-\frac{\Lambda_{\text {in }}}{3} R^{2}+\dot{R}^{2}}-\sigma_{\text {out }} R \sqrt{1-\frac{2 M G_{N}}{R}-\frac{\Lambda_{\text {out }}}{3} R^{2}+\dot{R}^{2}}=4 \pi \rho G_{N} R^{2} .
$$

In the above equation, $\Lambda_{\text {in, out }}=8 \pi G_{N} \epsilon_{\text {in, out }}$, are the cosmological constants representing the internal(in) and external(out) vacuum pressures; $\rho$ is the constant surface tension, and $\sigma_{\text {in }}\left(\sigma_{\text {out }}\right)= \pm 1$ depending on whether the radius of the bubble increases, or decreases, along the outward normal direction to the 2-brane surface embedded in the interior (exterior) metric. Here we have assumed, for the sake of simplicity, the membrane equation of state $\rho=-p=$ constant. However, we should emphasize that other equations of state, such as $p=0$ for dust, can be easily accomodated in our formalism. Following a suggestion first made in Ref. [4], we now propose to interpret Eq.(1.1) as the "Hamiltonian constraint ", $\mathcal{K}=0$, for a system described by the effective hamiltonian, or super-hamiltonian,

$$
\mathcal{K} \equiv 4 \pi \rho R^{2}-\sigma_{\text {in }} \frac{R}{G_{N}} \sqrt{1-\frac{\Lambda_{\text {in }}}{3} R^{2}+\dot{R}^{2}}+\sigma_{\text {out }} \frac{R}{G_{N}} \sqrt{1-\frac{2 M G_{N}}{R}-\frac{\Lambda_{\text {out }}}{3} R^{2}+\dot{R}^{2}}
$$

Then, we can use the Hamilton equation, $d \mathcal{K}=\dot{R} d P_{R}$, to deduce the momentum $P_{R}$ which is canonically conjugated to $\dot{R}$, and the corresponding lagrangian 


$$
\begin{aligned}
P_{R} & =\int \frac{\partial \mathcal{K}}{\partial \dot{R}} \frac{d \dot{R}}{\dot{R}} \\
L^{\mathrm{eff}} & =P_{R} \dot{R}-\mathcal{K}
\end{aligned}
$$

Thus, from the condition (1.1), we find

$$
P_{R}=\frac{R}{G_{N}} \ln \left|\left(\frac{\beta_{\text {out }}}{\beta_{\text {in }}}\right)^{1 / 2} \frac{\dot{R}+\sigma_{\text {in }} \sqrt{\dot{R}^{2}+\beta_{\text {in }}}}{\dot{R}+\sigma_{\text {out }} \sqrt{\dot{R}^{2}+\beta_{\text {out }}}}\right|
$$

where $\beta_{\text {in }} \equiv 1-\left(\Lambda_{\text {in }} / 3\right) R^{2}$ and $\beta_{\text {out }} \equiv 1-\left(\Lambda_{\text {out }} / 3\right) R^{2}-2 M G_{N} / R$.

The inverse Legendre transform (1.4) leads to the effective lagrangian we are looking for

$$
\begin{aligned}
L^{\mathrm{eff}} & =\frac{R}{G_{N}}\left(\sigma_{\text {in }} \sqrt{\dot{R}^{2}+\beta_{\text {in }}}-\sigma_{\text {out }} \sqrt{\dot{R}^{2}+\beta_{\text {out }}}-4 \pi \rho G_{N} R\right)+ \\
& -\frac{R \dot{R}}{G_{N}}\left[\sigma_{\text {in }} \sinh ^{-1}\left(\frac{\dot{R}}{\sqrt{\beta_{\text {in }}}}\right)-\sigma_{\text {out }} \sinh ^{-1}\left(\frac{\dot{R}}{\sqrt{\beta_{\text {out }}}}\right)\right] .
\end{aligned}
$$

From here, we deduce the proper time effective hamiltonian in canonical form,

$$
\mathcal{H}=4 \pi \rho R^{2}-\operatorname{sign}(\rho) \frac{R}{G_{N}}\left[\beta_{\text {in }}+\beta_{\text {out }}-2\left(\beta_{\text {in }} \beta_{\text {out }}\right)^{1 / 2} \cosh \left(\frac{G_{N} P_{R}}{R}\right)\right]^{1 / 2} .
$$

At this point, we should mention that the above Lagrangian and Hamiltonian can also be deduced, in a more fundamental way, directly from the Einstein-Hilbert action plus a boundary term, under the assumption of spherical symmetry. This can be verified, for instance, by extending the derivation of ref. [3] to our general case. The variational principle, in this case, leads precisely to the equations of motion derived from the Hamiltonian constraint $\mathcal{K}=0$. It is also worth observing that, by our reformulation, we have transformed the initial problem, i.e., the motion of a spherically symmetric self-gravitating membrane, into an equivalent, one dimensional, non-linear problem involving the dynamics of a single degree of freedom. In this " point-particle" interpretation of our effective lagrangian, one expects that " pair production " takes place, and in section II we discuss an explicit example of this process. Finally, we should mention that the formulation given in ref. [10] is conceptually close to ours, but is based on the use of the Schwarzschild coordinate time as evolution parameter along the bubble trajectory; the drawback of this choice of coordinate is that, even assuming a vanishing external pressure, it is impossible to Legendre transform the effective lagrangian and obtain the corresponding hamiltonian as a function of the canonical pair $\left(R, P_{R}\right)$.

Note that the hamiltonian (1.7) involves a square root operation in analogy to the familiar expression of the energy of a relativistic point particle. In our case the coefficient of the square root depends on sign $(\rho)$ in order to be consistent with the classical equation of motion (1.1). The opposite sign is classically meaningless. However, since we are dealing with a relativistic system, both positive and negative energies become physically relevant at the quantum level. This leads us to a broader interpretation of spacetime foam as a "Dirac sea of extended objects", in which not only wormholes, but also black holes and vacuum bubbles are continuously created and destroyed as zero-point energy fluctuations in 
the gravitational quantum vacuum. As a matter of fact, the effective lagrangian (1.6), (or the effective hamiltonian (1.7)), encodes the dynamics of a four-parameter $\left(\rho, \Lambda_{\text {in }}, \Lambda_{\text {out }}, M\right)$ family of spherically symmetric, classical solutions of the self-gravitating bubble equation of motion. Furthermore, the results (1.6) and (1.7) can be extended in a straightforward manner to an even larger family of solutions by endowing the internal geometry with a nonvanishing Schwarzschild mass term $M_{\mathrm{in}} G_{N} / R$, or the external geometry with an electric charge.

The spacetime foam models currently available in the literature focus essentially on the Schwarzschild metric (see, however, ref. [2]), and correspond to the sector $M>0$ of our family of classical solutions. For instance, in the sub-sector $\rho>0, \Lambda_{\text {in }}>0, \Lambda_{\text {out }}=0$, one finds the vacuum bubbles discussed in ref. [11]. In particular, we recall the type E trajectories with $M>M_{c r}$, listed in the same reference, because they give rise to babyuniverses connected through wormholes to the parent universe. The characteristics of those trajectories are instrumental to our discussion in Section IV.

Other types of "foam-like" solutions belong to the subsector $\Lambda_{\text {in }}=\Lambda_{\text {out }}=0$. They include the "surgical " Schwarzschild-Schwarzschild wormholes [3], and the "hollow" Minkowski-Schwarzschild wormholes [4], [12], while the region $\rho<0$ of the same subsector contains the traversable wormholes, i.e. wormholes whose throats can be crossed by timelike observers. In this connection, note that in our membrane model a negative tension plays the same role as the negative energy density of the more conventional wormholes made out of "dust ": it provides the "repulsive force" required to oppose the gravitational collapse of the throat [13]. The explicit correspondence between negative energy density

and surface tension is provided by the simple relation: $\rho \leftrightarrow m / 4 \pi R^{2}$. However, while the negative energy density of dusty wormholes is ascribed to some kind of exotic matter [13], or to gravitational vacuum polarization [14], we suggest to interpret membranes with negative tension as boundary layers between different physical vacua as suggested, for instance, by the existence of normal and confining vacua in QCD. More about negative energy density later.

In section II we show that in the flat spacetime limit, i.e. for $G_{N} \rightarrow 0$, the resulting lagrangian can be used to compute the false vacuum decay amplitude in ordinary quantum field theory.

In sections III and IV, as an explicit application of our general method, we shall study two examples of gravitational fluctuations in the sector $\Lambda_{i n}=\Lambda_{\text {out }}=M=0$, which correspond to vacuum bubbles. Their discussion and comparison with the existing literature on the subject provide an excellent testing ground for the validity of our approach.

\section{FALSE VACUUM DECAY}

Our effective approach is general enough to include bubble dynamics in the absence of gravity .As a matter of fact, the limit $G_{N} \rightarrow 0$ provides a necessary consistency check of our method and represents a special case worth investigating in and by itself.

The correct limiting procedure requires to express the two cosmological constants in terms of the corresponding vacuum energy densities $\epsilon_{\text {in }}, \epsilon_{\text {out }}: \Lambda_{\text {in }}=8 \pi G_{N} \epsilon_{\text {in }}, \Lambda_{\text {out }}=8 \pi G_{N} \epsilon_{\text {out }}$. In a single Minkowski domain $\sigma_{\text {in }}=\sigma_{\text {out }}=1$. Then, by expanding $L^{\text {eff }}$ up to the first order in 
$G_{N} / R^{2}$ we obtain,

$$
L^{\mathrm{eff}}=-4 \pi \rho R^{2}-\frac{4 \pi}{3} \Delta \epsilon R^{3} \sqrt{1+\dot{R}^{2}}, \quad \Delta \epsilon \equiv \epsilon_{\mathrm{in}}-\epsilon_{\mathrm{out}} .
$$

This effective lagrangian represents the minisuperspace approximation of the gauge action for the membrane

$$
\begin{aligned}
I= & -\rho \int d^{3} \xi \sqrt{-\gamma}-\frac{e}{3 !} \int d^{4} x J^{\mu \nu \rho} A_{\mu \nu \rho} \\
& -\frac{1}{2 \cdot 4 !} \int d^{4} x F_{\mu \nu \rho \sigma} F^{\mu \nu \rho \sigma}+\frac{1}{3 !} \int d^{4} x \partial_{\mu}\left(F^{\mu \nu \rho \sigma} A_{\nu \rho \sigma}\right),
\end{aligned}
$$

where $\gamma$ is the determinant of the induced metric on the 2-brane world tube $x^{\mu}=x^{\mu}\left(\xi^{a}\right)$, $a=0,1,2$, and $J^{\mu \nu \rho}(x)$ is the 2-brane current [9]. The last term in (2.2) is a total divergence ensuring that variations of the gauge potential $A_{\mu \nu \rho}$ will not produce unusual boundary terms due to the presence of the 2 -brane world tube. The corresponding hamiltonian is

$$
\begin{aligned}
\mathcal{H} & =4 \pi \rho R^{2}+\frac{4 \pi}{3} \Delta \epsilon R^{3} \sqrt{1-\left(3 P_{R} / 4 \pi \rho R^{3}\right)^{2}} \\
P_{R} & =-\frac{4 \pi}{3} \Delta \epsilon R^{3} \frac{\dot{R}}{\sqrt{1+\dot{R}^{2}}} .
\end{aligned}
$$

The classical trajectories describing true vacuum bubbles are solutions of the hamiltonian constraint $\mathcal{H}=0$, stating that the total mass energy of a vacuum bubble is vanishing. From equation (2.3) we see that classical solutions, corresponding to bubbles with positive surface tension, are allowed only if $\Delta \epsilon<0$, that is, the internal energy density ( of the true vacuum ) must be smaller than the external energy density ( of the false vacuum ), the net amount of energy released in the transition being converted into (positive) kinetic energy of the bubble wall. The semi-classical picture of the true vacuum domain nucleation corresponds to a classically forbidden motion. The classically unphysical tunneling trajectory is a solutions of the euclidean, equation of motion obtained via the Wick rotation $\tau_{E} \equiv i \tau ; P_{E} \equiv i P$

$$
\mathcal{H}_{E}=0, \quad \Rightarrow P_{E}=4 \pi \rho R^{2}\left(1-\frac{R^{2}}{R_{0}^{2}}\right)^{1 / 2}, \quad R_{0}=\frac{3 \rho}{\Delta \epsilon} .
$$

Then, using the classical solution (2.5) for $P_{E}$ in the WKB integral for the calculation of the nucleation probability through tunnel effect, we obtain the standard result,

$$
B=2 \int_{0}^{R_{0}} d R P_{E}(R)=8 \pi \rho \int_{0}^{R_{0}} d R R^{2}\left(1-\frac{R^{2}}{R_{0}^{2}}\right)^{1 / 2}=\frac{\pi^{2}}{2} \rho R_{0}^{3},
$$

in agreement with the Coleman-De Luccia $B$ decay coefficient [18].

\section{III. " MINKOWSKI PAIR" CREATION}

The simplest example of gravitational vacuum fluctuation is given by the spontaneous nucleation of a vanishing mass-energy shell in Minkowski spacetime. Despite the triviality 
of both the internal and external geometries, the dynamics of such a process is nonetheless affected by the unavoidable ambiguities and technical problems of quantum gravity. To circumvent all these difficulties, one usually employs a semi-classical model obtained by gluing together two Minkowski metrics along the bubble trajectory .

In our approach, the steps are as follows. The signs of $\sigma_{\text {in }}, \sigma_{\text {out }}$ are fixed by the matching condition

$$
\sigma_{\text {in }} R \sqrt{1+\dot{R}^{2}}-\sigma_{\text {out }} R \sqrt{1+\dot{R}^{2}}=4 \pi \rho G_{N} R^{2}
$$

For a positive surface tension we have $\sigma_{\text {in }}=-\sigma_{\text {out }}=1$, otherwise the two spacetime domains cannot be glued together. The resulting geometry represents the limiting configuration of vanishing cosmological constant and Schwarzschild mass discussed in appendix D of ref. [11]. This is a closed universe formed by two compact spherical regions of flat spacetime. The shell equation of motion obtained by squaring (3.1) is

$$
\dot{R}^{2}=-1+4 \pi^{2} \rho^{2} G_{N}^{2} R^{2}
$$

and admits only bounce solutions irrespective of the sign of $\rho$. In our case

$$
\begin{gathered}
L^{\mathrm{eff}}=-4 \pi \rho R^{2}+\frac{2 R}{G_{N}} \sqrt{1+\dot{R}^{2}}-\frac{2 R \dot{R}}{G_{N}} \sinh ^{-1} \dot{R}, \\
P_{R}=\frac{\partial L^{\mathrm{eff}}}{\partial \dot{R}}=-\frac{2 R}{G_{N}} \sinh ^{-1} \dot{R}, \\
\mathcal{H}=4 \pi \rho R^{2}-\frac{2 R}{G_{N}} \cosh \left(\frac{G_{N} P_{R}}{2 R}\right) .
\end{gathered}
$$

Note that, in order to recover Eq. (3.5) from the general formula (1.7), one must carefully assign the phases of the complexified functions $\sqrt{\beta_{\text {in }}}, \sqrt{\beta_{\text {out }}}$ in the analytically extended Schwarzschild manifold, and only then one may consider the limit of vanishing Schwarzschild mass. This is because the effective hamiltonian (1.7) is actually a complex function when considered on the maximally analytic extension of the underlying spacetime manifold. Note, also, that the kinetic term, obtained by expanding " cosh" up to second order, is negative. Therefore, $\mathcal{H}=0$ is the classical equation of motion of a (positive kinetic energy) particle in the reversed potential $V(R) \sim-4 \pi \rho R^{3}+2 R^{2} / G_{N}$. This potential constitutes an effective barrier for the "particle" motion and explains the absence of a discrete spectrum of stationary quantum states. The classical dynamics of this type of domain wall has been discussed in ref. [15] with special emphasis on the repulsive character of the resulting gravitational field. In our formulation, this repulsive effect is plainly exhibited by the potential above. We propose to associate the expected quantum mechanical "leakage " through the potential barrier with the process of "universe creation" by quantum tunneling from nothing. In order to give substance to this interpretation, one needs to compute the corresponding transmission coefficient, and, in order to perform this calculation, we rotate the dynamical quantities to imaginary time: 


$$
P_{E}=\frac{2 R}{G_{N}} \cos ^{-1}\left(\frac{R}{R_{G}}\right), \quad \text { where } \quad R_{G}=\frac{1}{2 \pi \rho G_{N}} .
$$

This gives, for the nucleation coefficient

$$
B=\frac{4}{G_{N}} \int_{0}^{R_{G}} d R R \cos ^{-1}\left(\frac{R}{R_{G}}\right)=\frac{1}{8 \pi \rho^{2} G_{N}^{3}},
$$

in agreement with the bounce calculation of ref. [12].

In the case of negative surface tension the calculation proceeds along the same steps outlined above, but with $\sigma_{\text {in }}=-1, \sigma_{\text {out }}=+1$. The resulting geometry is the limiting case of a "surgically" constructed Schwarzschild wormhole, when the mass is sent to zero [3]. However, any constant time section of the resulting spacetime has infinite volume. Thus, this second type of "Minkowski pair" does not correspond to a compact object and cannot be nucleated quantum mechanically from "nothing".

\section{INFLATIONARY BUBBLE NUCLEATION AMPLITUDE}

The next case study is somewhat more subtle and we discuss it to illustrate the applicability of our effective method. To the extent that this system may arise as a fluctuation of the gravitational vacuum, we interpret it as a possible constituent of the Planckian spacetime foam even though it does not correspond to any class of wormholes. What we have in mind is the nucleation of a false vacuum de Sitter bubble in a Minkowski background which represents the key mechanism proposed in ref. [11], and expanded in ref. [10], to generate quantum mechanically an inflationary domain. From our vantage point, the difficulty of that proposal is the presence of a virtual black hole, decaying through Hawking radiation, as an intermediate state between the initial Minkowski state and the final Minkowski+de Sitter state. This intermediate state involves the still obscure issue of the final stage of black hole evaporation. Interestingly enough, it is possible to bypass this difficulty by choosing a negative surface tension and vanishing total mass energy for the original quantum fluctuation that triggers the process in the first place. Physically, this assumption of negative surface tension may be justified by a simple analogy with the multiphase vacuum of QCD. If the unified field theory undergoing primordial phase transitions is of the Ginzburg-Landau type, then, for some choice of the coupling constants, bags can form around test charges with positive volume and negative surface energy [16]. The sign of the surface tension follows from the negative condensation energy. The vacuum state for such a model behaves as a type II superconductor with maximal boundary surface between the normal ( non-confining ) phase and the ordered ( confining ) phase [17].

With the above choice of surface tension and vanishing total mass-energy, the initial and final states are degenerate in energy and a spontaneous transition between them is allowed without an intermediate blackhole state.

Some preliminary remarks on the classical dynamics of a de Sitter bubble will be helpful in order to clarify our final result. The classical equation of motion for the bubble trajectory is 


$$
\begin{aligned}
\dot{R}^{2} & =-1+\frac{R^{2}}{R_{B}^{2}}, \\
R_{B} & =\frac{8 \pi|\rho| G_{N}}{16 \pi^{2} \rho^{2} G_{N}^{2}+1 / H^{2}}=\frac{R_{0}}{1+R_{0}^{2} / 4 H^{2}}
\end{aligned}
$$

where $H$ is the radius of the de Sitter cosmological event horizon, and $R_{0}=3|\rho| / \epsilon_{i n}$ is the nucleation radius in the absence of gravity. Equation (4.1) admits only a bounce-solution which represents an infinite domain of de Sitter vacuum collapsing down to a nonvanishing minimum radius $R_{B}<R_{0}$, and then re-expanding indefinitely. Note also that $R_{B} \leq H$, and that the equality holds only if the surface tension and the internal vacuum energy density are tuned so that $\epsilon_{i n}=6 \pi \rho^{2} G_{N}$.

Having assumed a negative surface tension, we retrace our steps as in the previous case study: the matching condition now is

$$
\sigma_{\text {in }} R \sqrt{1+\dot{R}^{2}-R^{2} / H^{2}}-\sigma_{\text {out }} R \sqrt{1+\dot{R}^{2}}=4 \pi \rho G_{N} R^{2}, \quad H^{2} \equiv 3 / \Lambda_{\text {in }},
$$

and gives us

$$
\begin{aligned}
\sigma_{\text {out }} & =+1, \\
\sigma_{\text {in }} & =-\operatorname{sign}\left(16 \pi^{2} \rho^{2} G_{N}^{2}-\frac{1}{H^{2}}\right)=-\operatorname{sign}\left(R_{0}-2 H\right) .
\end{aligned}
$$

Equations (4.4) and (4.5) show that, while the sign of $\sigma_{\text {out }}$ is fixed to +1 along the bubble trajectory, the sign of $\sigma_{i n}$ depends on the relative size of $R_{0}$ and $2 H$. The two nucleation modes actually correspond to two different Penrose diagrams, but we shall omit their discussion in this brief communication.

Presently, for reasons of clarity and conciseness, we choose to discuss the case $R_{0}<2 H \rightarrow \sigma_{i n}=+1$. The corresponding semi-classical solution, which describes the nucleation of an expanding de Sitter bubble, is obtained by matching the expanding half of the classical bounce to a quantum tunneling solution. Then, the classical turning point acquires the meaning of nucleation radius. The corresponding lagrangian and hamiltonian are,

$$
\begin{aligned}
L^{\mathrm{eff}} & =\frac{R}{G_{N}}\left(\sqrt{\beta_{i n}+\dot{R}^{2}}-\sqrt{1+\dot{R}^{2}}-4 \pi \rho G_{N} R\right)+ \\
& -\frac{R \dot{R}}{G_{N}}\left[\sinh ^{-1}\left(\frac{\dot{R}}{\sqrt{\beta_{\text {in }}}}\right)-\sinh ^{-1} \dot{R}^{2}\right], \\
P_{R} & =-\frac{R}{G_{N}}\left[\sinh ^{-1}\left(\frac{\dot{R}}{\sqrt{\beta_{\text {in }}}}\right)-\sinh ^{-1} \dot{R}\right] \\
\mathcal{H} & =4 \pi \rho R^{2}+\frac{R}{G_{N}}\left[\beta_{\text {in }}+1+2 \sqrt{\beta_{\text {in }}} \cosh \left(\frac{G_{N} P_{R}}{R}\right)\right]^{1 / 2} .
\end{aligned}
$$

Note that we have fixed the phase of $\sqrt{\beta_{\text {in }}}$ and $\sqrt{\beta_{\text {out }}}$ by the condition that the above hamiltonian coincides with the hamiltonian (3.5) in the limit of vanishing internal energy density. The classical equation $\mathcal{H}=0$, gives 


$$
P_{R}=\frac{R}{G_{N}} \cosh ^{-1}\left[\frac{\left(4 \pi \rho G_{N} R\right)^{2}-1-\beta_{i n}}{2 \sqrt{\beta_{i n}}}\right] .
$$

This enables us to evaluate the tunneling amplitude

$$
B=2 \int_{0}^{R_{B}} d R\left|P_{E}(R)\right|=-\frac{1}{G_{N}} \int_{0}^{R_{B}} d R R^{2} \frac{d}{d R} \cos ^{-1}\left[\frac{\left(4 \pi \rho G_{N} R\right)^{2}-1-\beta_{\text {in }}}{2 \sqrt{\beta_{\text {in }}}}\right],
$$

and an explicit calculation yields

$$
\begin{aligned}
B & =4 \pi|\rho| \int_{0}^{R_{B}} d R R^{2}\left(1-\frac{R^{2}}{H^{2}}\right)^{-1}\left(1-\frac{R^{2}}{R_{B}^{2}}\right)^{-1 / 2}\left(\frac{R^{2}}{4 \pi|\rho| G_{N} R_{B} H^{2}}-1\right) \\
& =\frac{\pi^{2}|\rho|}{2} R_{B}^{2} R_{0} .
\end{aligned}
$$

Our last comment concern the physical interpretation of this result. The model discussed above describes the quantum birth of an inflationary bubble in a Minkowski background. In connection with this process, we find that there is a lingering ambiguity in the published literature. It is indeed interesting, and perhaps somewhat puzzling, that the initial radius and the nucleation rate in this case are the same as for the false vacuum decay, that is, the nucleation of a Minkowski bubble in a De Sitter background, originally discussed by Coleman and De Luccia [18]. With hindsight, this coincidence is hardly surprising since the two cases appear to be completely symmetrical due to the fact that the euclidean trajectory interpolating between the two vacuum states is the same in both cases. However, there is a difference, even at the classical level, and it lies in the global structure of the spacetime manifold in the two cases. The point is that, for an inflationary bubble in a Minkowski background, at a given instant, say the nucleation Minkowski time, all the points in the interval $0 \leq r \leq R_{B}$ suddenly undergo a phase transition from the Minkowski to the de Sitter geometry. Then, the new vacuum domain, driven by the negative pressure of the false vacuum, expands exponentially, eventually filling up the whole spacetime. In contrast, a true vacuum bubble, no matter how large, will never fill up the whole de Sitter manifold. As a matter of fact, in this difference lies the problem of the "graceful exit " from the inflationary stage [19]. 


\section{REFERENCES}

[1] A.Linde, Lectures on Inflationary Cosmology SU-ITP-94-36, Oct.1994 hep-th@xxx.lanl.gov-9410082

[2] I.H. Redmount, W. Suen, Phys. Rev. D47, (1993) R2163; D49, (1994) 5199.

[3] M.Visser, Phys. Rev. D43, (1991) 402

[4] V.A.Berezin, N.G.Kozimirov, V.A.Kuzmin, I.I.Tkachev, Phys. Lett. B212, (1988), 415

[5] V.A.Berezin, On a Quantum Mechanical Model of a Black Hole, UPR 176-CNRS Observatoire de Paris-Meudon, 92195, Meudon, France, (1990)

[6] M.Visser, Phys. Rev. D39, (1989) 3182; D41, (1990) 1116

Nucl. Phys. B328, (1989), 203

[7] J.A. Wheeler, Ann. Phys. (NY) 2, (1957) 604

[8] A.Aurilia, A.Smailagic, E.Spallucci, Phys Rev D51, (1995), 4410

[9] A.Aurilia, R.S.Kissack, R.Mann, E.Spallucci, Phys. Rev. D35, (1987), 2961

A.Aurilia, M.Palmer, E.Spallucci, Phys. Rev. D40, (1989), 2511

[10] E.Farhi, A.H.Guth, J.Guven, Nucl. Phys. B339, (1990), 417

[11] S.K.Blau, E.I.Guendelman, A.H.Guth, Phys. Rev. D35, (1987) 1747

[12] V.A.Berezin, V.A.Kuzmin, I.I.Tkachev, Phys. Lett. B207, (1988), 397

[13] M.S.Morris, K.S.Thorne Am. J. Phys. 56, 395, (1988)

[14] T.S.Roman Phys. Rev. D33, 3528, (1986)

[15] J.Ipser, P.Sikivie, Phys. Rev. D30, (1984) 712

[16] J.Hosek, Phys. Lett. B226, 377, (1989); Czech. J. Phys. 41, 1037, (1991)

[17] In this connection, see the accompanying memorial article in honor of Umezawa: S.Ansoldi, A.Aurilia. E.Spallucci, Membrane vacuum as a type-II superconductor, International Journal of Modern Physics B, (1996), in press.

[18] S.Coleman, F.De Luccia, Phys. Rev. D21, (1980) 3305

[19] A.H.Guth, E.J.Weinberg, Nucl. Phys. B212, (1983), 321 